\title{
Can peritumoral regions increase the efficiency of machine- learning prediction of pathological invasiveness in lung adenocarcinoma manifesting as ground-glass nodules?
}

\author{
Xiang Wang ${ }^{1 \#}$, Kaili Chen ${ }^{2 \#}$, Wei Wang ${ }^{1,3 \#}$, Qingchu $\mathrm{Li}^{1}$, Kai Liu ${ }^{1}$, Qianyun $\mathrm{Li}^{4}$, Xing Cui ${ }^{5}$, Wenting Tu${ }^{1}$, \\ Hongbiao Sun ${ }^{1}$, Shaochun Xu ${ }^{1}$, Rongguo Zhang ${ }^{5}$, Yi Xiao ${ }^{1}$, Li Fan ${ }^{1}$, Shiyuan Liu ${ }^{1}$ \\ ${ }^{1}$ Department of Radiology, Changzheng Hospital, Naval Medical University, Shanghai, China; ${ }^{2}$ Department of Hematology, The Myeloma \& \\ Lymphoma Center, Changzheng Hospital, Naval Medical University, Shanghai, China; ${ }^{3} 71282$ Hospital, Baoding, China; ${ }^{4}$ Department of Radiology, \\ Taizhou Hospital of Zhejiang Province, Linhai, China; ${ }^{5}$ Beijing Infervision Technology Co. Ltd., Beijing, China \\ Contributions: (I) Conception and design: X Wang, K Chen, W Wang; (II) Administrative support: X Cui, R Zhang, S Liu; (III) Provision of study \\ materials or patients: X Wang, Q Li; (IV) Collection and assembly of data: K Liu, W Tu, H Sun, S Xu; (V) Data analysis and interpretation: K Chen, \\ Y Xiao, L Fan; (VI) Manuscript writing: All authors; (VII) Final approval of manuscript: All authors. \\ \#These authors contributed equally to this work. \\ Correspondence to: Li Fan, MD; Shiyuan Liu, MD. Department of Radiology, Changzheng Hospital of the Naval Medical University, No. 415 \\ Fengyang Road, Shanghai 200003, China. Email: czyxfl@smmu.edu.cn; liushiyuan@smmu.edu.cn.
}

Background: The peri-tumor microenvironment plays an important role in the occurrence, growth and metastasis of cancer. The aim of this study is to explore the value and application of a CT image-based deep learning model of tumors and peri-tumors in predicting the invasiveness of ground-glass nodules (GGNs).

Methods: Preoperative thin-section chest CT images were reviewed retrospectively in 622 patients with a total of 687 pulmonary GGNs. GGNs are classified according to clinical management strategies as invasive lesions (IAC) and non-invasive lesions (AAH, AIS and MIA). The two volumes of interest (VOIs) identified on CT were the gross tumor volume (GTV) and the gross volume of tumor incorporating peritumoral region (GPTV). Three dimensional (3D) DenseNet was used to model and predict GGN invasiveness, and five-fold cross validation was performed. We used GTV and GPTV as inputs for the comparison model. Prediction performance was evaluated by sensitivity, specificity, and area under the receiver operating characteristic curve (AUC).

Results: The GTV-based model was able to successfully predict GGN invasiveness, with an AUC of 0.921 (95\% CI, 0.896-0.937). Using GPTV, the AUC of the model increased to 0.955 (95\% CI, 0.939-0.971).

Conclusions: The deep learning method performed well in predicting GGN invasiveness. The predictive ability of the GPTV-based model was more effective than that of the GTV-based model.

Keywords: Pulmonary adenocarcinoma; deep learning; tumor invasiveness; peritumoral region; X-ray computed tomography (X-ray CT)

Submitted Sep 26, 2020. Accepted for publication Dec 18, 2020.

doi: $10.21037 /$ jtd-20-2981

View this article at: http://dx.doi.org/10.21037/jtd-20-2981 


\section{Introduction}

Early detection of lung cancer is gradually increasing with a wider application of low-dose CT screenings and increasing personal health awareness, especially in patients with lung adenocarcinoma in ground-glass nodules (GGNs) $(1,2)$. The standard 2015 international multidisciplinary classification for lung adenocarcinoma outlines four categories of lung adenocarcinoma: atypical adenomatous hyperplasia (AAH), adenocarcinoma in situ (AIS), minimally invasive adenocarcinoma (MIA), and invasive adenocarcinoma (IAC). The 5 -year disease-free survival rate is almost $100 \%$ for patients diagnosed with preinvasive lesions (AAH and AIS) and MIA with a sub-lobar resection (3-5). IAC treatment mainly consists of lobectomy with a five-year survival rate after surgery under $90 \%(6,7)$. Thus, accurate preoperative identification of the degree of invasiveness is crucial for predicting the prognosis of GGNs and guiding proper surgical treatment.

Preoperative CT features play a critical role in assessing the invasiveness of GGNs, however the CT features of early lung cancer are often atypical $(8,9)$. In recent years, traditional computer-aided technology and radiomics have aided the evaluation of lung adenocarcinoma invasiveness $(10,11)$. However, these two methods still have some limitations, including the need for hand-engineered feature extraction from images, poor repeatability and reliability, and time-consuming and cumbersome workflows. Artificial intelligence (AI) and deep learning show promise for broad application in medicine $(12,13)$. Convolutional neural networks (CNNs), in particular, have been effective in detecting and classifying lesions $(14,15)$.

Previous studies have largely focused on the relationship between primary tumor volumes and tumor invasiveness, while neglecting the influence of the peri-tumor microenvironment. However, Valencia et al. (16) found that the cells and tissues around the tumor are closely related to the occurrence, growth and metastasis of cancer. A change in the peritumoral microenvironment might influence poor prognosis in patients with malignant tumors. Zhang et al. (17) found that the density of the peritumoral lymphatic microvessel might provide a reference for regional lymph node (LN) metastasis in non-small cell lung cancer (NSCLC). The microenvironment has also been reported to secrete growth factors and cytokines that induce hypoxia and angiogenesis, influencing the occurrence, development and metastasis of NSCLC (18). The peritumoral microenvironment is also important for evaluating the malignant biological behavior of tumors, not just the tumor itself $(16,19,20)$. Thus, we speculated that characteristics of the peritumoral tissue could be helpful in evaluating the invasiveness of GGNs. We propose that peritumoral characteristics may influence the accuracy of classification. Therefore, studying the role of image features on peritumoral lung parenchyma may be significant in evaluating invasiveness.

We developed a CNN-based model to predict the invasiveness of GGNs by extracting the gross tumor volume (GTV) and the gross volume of tumor incorporating peritumoral region (GPTV) from preoperative CT images to train and validate the model. We aimed to establish an effective, automatic and non-invasive prediction model based on preoperative CT images to guide early diagnosis and timely-intervention in GGNs. We present the following article in accordance with the MDAR reporting checklist (available at http://dx.doi.org/10.21037/jtd-20-2981).

\section{Methods}

\section{Study population}

All procedures performed in this study were in accordance with the Declaration of Helsinki (as revised in 2013) and approved by the Ethics Committee of the Changzheng Hospital, Naval Medical University (No. 2018SL049), and individual consent for this retrospective analysis was waived. Patient inclusion criteria were as follows: patients with a maximum GGN tumor diameter of $\leq 30 \mathrm{~mm}$ on thin-section CT, those with a pure ground-glass nodule (pGGN) or a mixed groundglass nodule (mGGN), patients with $0.625-1 \mathrm{~mm}$ thin sliced CT images that were obtained within the 2 weeks leading up to the operation, those with no previous therapy history, and those with a definite pathological diagnosis. The exclusion criteria were as follows: patients without a thin-slice image, those with CT image artifacts or unclear GGN boundaries and those with incomplete clinical data. From December 2012 to September 2018, a total of 622 patients (median age 57 years, 417 women) who met the criteria were included. These patients had a total of 687 GGNs, of which 311 were invasive and 376 were noninvasive.

All patients with GGNs were evaluated using one of five multidetector CT scanners (manufacturer: Toshiba, General Electric or Philips) with the following settings: tube voltage, $120 \mathrm{kVp}$; tube current, $50-150 \mathrm{~mA}$; image 
Table 1 The scanning parameters on CT for the patients with GGNs

\begin{tabular}{|c|c|c|c|c|c|}
\hline Parameters & \multicolumn{5}{|c|}{ Manufacture } \\
\hline Tube voltage (Kv) & 120 & 120 & 120 & 120 & 120 \\
\hline Effective power of tube (mA) & $50-150$ & $50-150$ & Auto $\mathrm{mA}$ & $50-150$ & $50-150$ \\
\hline Detector collimation (mm) & $16 \times 0.5$ & $64 \times 0.625$ & $64 \times 0.625$ & $64 \times 0.625$ & $128 \times 0.625$ \\
\hline Slice thickness $(\mathrm{mm})$ & 1.000 & 1.000 & 1.000 & 1.000 & 0.625 \\
\hline Number of cases & 30 & 48 & 72 & 103 & 248 \\
\hline
\end{tabular}

$\mathrm{CT}$, computed tomography; GGN, ground-glass nodule.

matrix, $512 \times 512$ pixels; and a 0.5 second scanning duration. Images were reconstructed using a standard algorithm reconstruction with a thickness of $0.625-1 \mathrm{~mm}$. All patients were scanned in the supine position and at full inspiration, as measured from the thoracic entrance to the base of the lungs. CT parameters are shown in Table 1. All pathological diagnoses were identified from pulmonary pathology by pathologists, especially for patients with multiple nodules. The pathological subtypes of each GGN were categorized according to the IASLC/ATS/ERS classification of lung adenocarcinoma in 2015 (21). The following clinical data were recorded for all patients: age, sex, smoking history (never, current, or former), family history, and occurrence of chronic obstructive pulmonary disease (COPD). Characteristics of the peritumoral region were described from CT features, including margin characteristics (smooth, lobular or spiculated), pleural indentation, nodule-lung interface (well defined or ill defined), and prevalence of emphysema in the peritumoral region. The demographic and clinical characteristics of all patients are presented in Table 2 and Table S1.

\section{Nodule segmentation and labeling}

The primary GGN lesion area was manually identified and labeled by a radiologist (with 4 years of experience in chest CT diagnosis), and confirmed by another pulmonary radiologist (with 7 years of experience in chest CT diagnosis). Neither of the radiologists knew the relevant clinicopathological information of the patients. Final consensus was reached by group discussion if there were different interpretations between the radiologists. The nodule segmentation was determined in every $2-\mathrm{D}$ CT slice with lung window settings (window width, 1,500
$\mathrm{HU}$; window level, -450 HU) and mediastinal window settings (width, $300 \mathrm{HU}$; window level, $60 \mathrm{HU}$ ) using an open-source Medical Imaging Interaction Toolkit (MITK) software (version 2017.07; http://www.mitk.org/). The methods for evaluating the reproducibility of the region of interest (ROI) have been previously validated (20).

During labeling, GGNs were drawn manually along the lesion edge with the largest lesion area, including the ground-glass component as defined by the ROI. The volume of the ROI was defined from the CT scan as the GTV. Large vessels and pulmonary arteries were excluded when marking. The volume of the GTV region was defined according to previous methods (20) as the area that evenly expanded in three directions as the PTV. Similarly, the GTV and PTV merged to become the GPTV.

All CT scans were rescaled to a voxel size of $1 \times 1 \times 1 \mathrm{~mm}^{3}$ using linear interpolation, and then all GTVs and GPTVs were extracted from the resampled images. The PTV in the GPTV was generated with a morphology processing method called dilation operation, which is setting the increasing size and expanding the surrounding pixels of the GTV within this size. The increasing size of the peritumor region was set to 5 pixels, i.e., $5 \mathrm{~mm}$ according to methods in Wu et al. and Beig et al. $(22,23)$ (shown in Figure 1).

\section{Data preprocessing}

To establish a cohort, we retrospectively collected data from 622 patients between December 2012 and September 2018, and selected 687 total GGNs to analyze from these patients. All nodules were diagnosed pathologically and resected specimens were categorized according to the 2015 IASLC/ ATS/ERS classification system (21). Four types of specimens were collected, including 113 AAH, 148 AIS, 115 MIA, and 
Table 2 Demographic data for patients

\begin{tabular}{|c|c|c|}
\hline Characteristics & Number & Percentage \\
\hline Numbers of patients & 622 & - \\
\hline Age (y), median [range] & 57 [27-87] & - \\
\hline \multicolumn{3}{|l|}{ Gender } \\
\hline Male & 205 & $32.96 \%$ \\
\hline Female & 417 & $67.04 \%$ \\
\hline Smoking history & 116 & $18.65 \%$ \\
\hline Patients with family history & 17 & $2.73 \%$ \\
\hline Patients with COPD & 35 & $5.63 \%$ \\
\hline Numbers of nodule & 687 & - \\
\hline \multicolumn{3}{|l|}{ Pathology } \\
\hline AAH & 113 & $16.45 \%$ \\
\hline AIS & 148 & $21.54 \%$ \\
\hline MIA & 115 & $16.74 \%$ \\
\hline IAC & 311 & $45.27 \%$ \\
\hline \multicolumn{3}{|c|}{ Tumor diameter (mm), median [range] } \\
\hline AAH & $11[9-13]$ & - \\
\hline AIS & $11[10-13]$ & - \\
\hline MIA & $13[11-16]$ & - \\
\hline IAC & 18 [15-22] & - \\
\hline Density (pGGNs) & 435 & $63.32 \%$ \\
\hline $\mathrm{AAH}$ & 106 & $24.37 \%$ \\
\hline AIS & 134 & $30.80 \%$ \\
\hline MIA & 88 & $20.23 \%$ \\
\hline IAC & 107 & $24.60 \%$ \\
\hline Density (mGGNs) & 252 & $36.68 \%$ \\
\hline AAH & 7 & $2.78 \%$ \\
\hline AIS & 14 & $5.56 \%$ \\
\hline MIA & 27 & $10.71 \%$ \\
\hline IAC & 204 & $80.95 \%$ \\
\hline
\end{tabular}

$\mathrm{AAH}$, atypical adenomatous hyperplasia; AIS, adenocarcinoma in situ; MIA, minimally invasive adenocarcinoma; IAC, invasive adenocarcinoma; pGGN, pure ground-glass nodules; mGGNs, mixed ground glass nodules; COPD, chronic obstructive pulmonary disease.
311 IAC specimens. Patients diagnosed with AAH/AIS/ MIA were eligible for sub-lobar resection, and the diseasefree survival rate after surgery was close to $100 \%$, which is significantly better than invasive lung adenocarcinoma (38-86\%) (6). Here, the judgment of pulmonary nodule invasiveness was regarded as a binary classification. AAH, AIS, and MIA were classified as type 1, non-invasive lesions; IAC was classified as type 0 , invasive lesions.

All VOIs were pre-processed before inputting them into the model. The pre-processing included two steps. First, all VOIs were resized to $64 \times 64 \times 64$ voxels. Then, all voxel values in the 3 dimensional data were normalized to a unified size $(-1,1)$.

\section{Deep learning system}

We developed a 3D DenseNet to predict GGN invasiveness. The proposed network is shown in Figure 2. The network structure contains four dense blocks and three transition blocks. Each dense block is composed of a series of $1 \times 1 \times 1$ convolutional layers, $3 \times 3 \times 3$ convolutional layers, and skip connections. Each transition block includes a $1 \times 1 \times 1$ convolutional layer for reducing network parameters and a $2 \times 2 \times 2$ average pooling operation with stride 2 for down-sampling. A fully connected layer followed by a dropout layer with drop ratio of 0.5 was used in our network. The softmax function was used to output the probability of each class. The final classification made by the model was determined by assigning the class with the highest probability.

Five-fold validation was applied to train and validate the prediction model. To speed up network convergence, model parameters were pre-trained on the ImageNet before the fully connected layers were frozen. The whole network was optimized by the Adam optimizer. The initial learning rate was 0.001 .

\section{Model evaluation, statistic analysis, and software}

To evaluate the model's predictive capability, the probability of each 3D data output was compared with the corresponding label. The prediction performance was evaluated using sensitivity (SEN), specificity (SPE), and area 

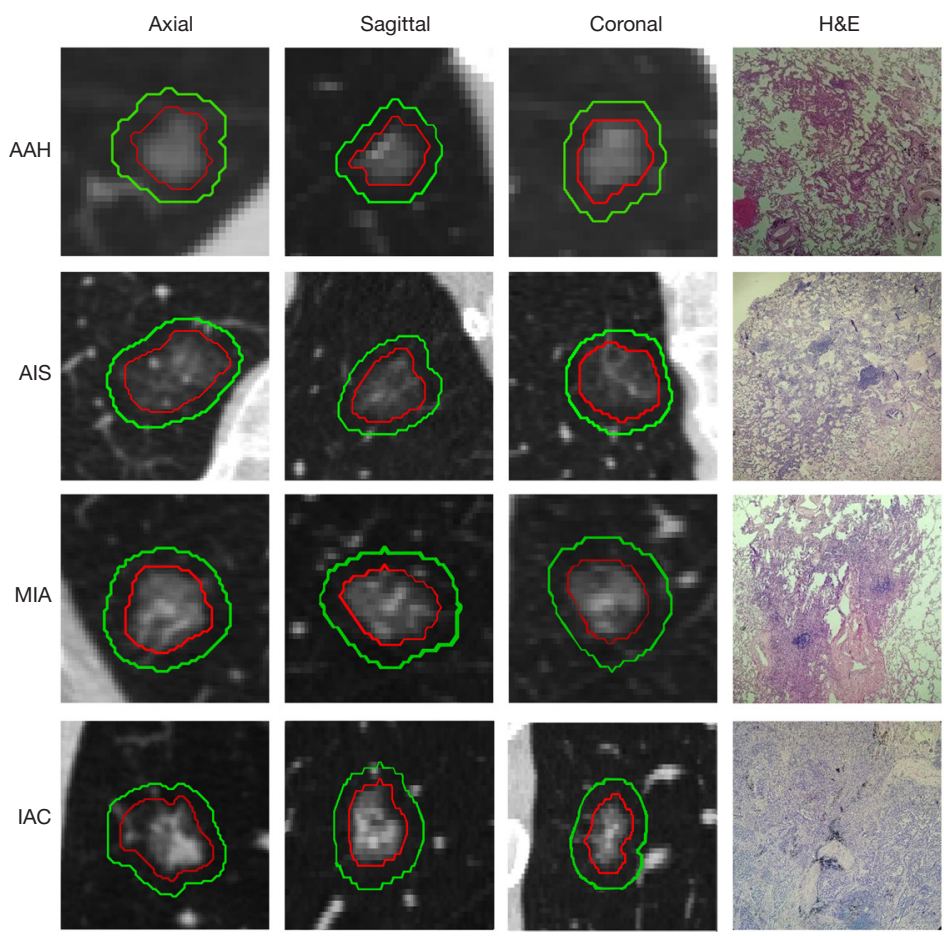

Figure 1 Examples in the dataset of nodule patches in axial, sagittal, and coronal views. The red contours represent the gross tumor volume (GTV), and the green contours represent the peritumoral volume (PTV) from four patients. H\&E, hematoxylin and eosin stain. Magnification, $\times 40$.
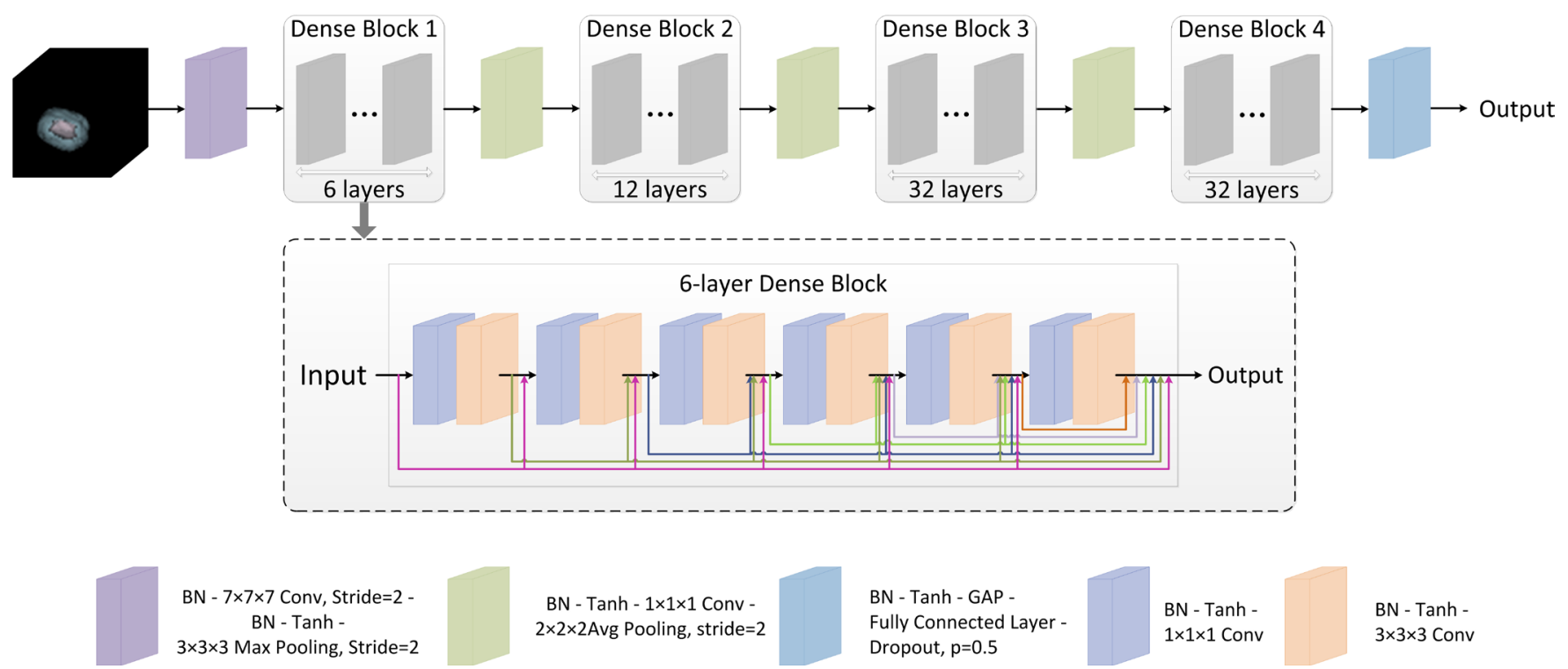

BN - Tanh - GAP Fully Connected Layer Dropout, $\mathrm{p}=0.5$

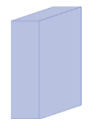

BN - Tanh $1 \times 1 \times 1$ Conv

BN - Tanh $3 \times 3 \times 3$ Conv

Figure 2 The architecture of the DenseNet used in our study. 
under the receiver operating characteristic curve (AUC). The positive predictive value (PPV), the negative predictive value (NPV), and accuracy (ACC) were also used to assess model performance.

Continuous variables are generally expressed as means and ranges, and categorical variables are represented as numbers and proportions. The DeLong test was used for statistical comparison of the ROC curves (24). P values $<0.05$ were considered significant.

A software based on an Ubuntu 18.04 operating system with the deep learning toolkit called MXNet was used to build the models. Cross validation was run on the NVIDIA GeForce GTX 1080 GPU (NVIDIA, Beijing, China) and all processing was performed in the Python 3.6 environment. Statistical analysis was performed using $\mathrm{R}$ software (version 3.5.1, https://www.r-project.org/).

\section{Results}

Table 1 lists the histological distribution of GGNs. Throughout the observation period, a total of 687 nodules with GGN components were detected on thin-section CT images in 622 patients. Among these 687 GGNs, 113 were defined as AAH, 148 were AIS, 115 were MIA, and 311 were IAC. Most patients with GGNs were identified in women $(\mathrm{n}=417,67.04 \%)$, nonsmokers $(\mathrm{n}=506,81.35 \%)$, and $435(63.32 \%)$ GGNs were classified as pGGNs. The average patient age was 57 years (range, 27-87 years), 17 patients had a family history of lung cancer and 35 patients had COPD. We also studied peritumoral CT features. We found no significant intergroup differences in peritumoral emphysema $(\mathrm{P}=0.072)$. However, there were significant differences in margin $(\mathrm{P}<0.001)$, nodule-lung interface $(\mathrm{P}=0.035)$, pleural indentation $(\mathrm{P}<0.001)$ between these two groups. These data are summarized in Table S1. Table 3 shows our CNN model's performance using two input types. The CNN model performed well by using both GTV and GPTV. The GTPV all improved in each fold, though only Fold 3 existed statistically significant (DeLong's test: $\mathrm{P}=0.014)$. Overall, the mean AUC, accuracy, sensitivity, specificity, PPV and NPV of the GTV-trained model were 0.921 (95\% CI: 0.896-0.937), 0.839 (95\% CI: 0.812-0.868), 0.800 (95\% CI: $0.759-0.843$ ), 0.899 (95\% CI: $0.851-$ 0.924), 0.897 (95\% CI: 0.863-0.927), and 0.787 (95\% CI: $0.745-0.832)$, respectively. The same indexes of the GPTVtrained model were 0.955 (95\% CI: $0.939-0.971), 0.904$ (95\% CI: 0.881-0.927), 0.893 (95\% CI: 0.861-0.925), 0.917 (95\% CI: 0.884-0.947), 0.929 (95\% CI: 0.901-0.955), and
0.876 (95\% CI: 0.841-0.912), respectively. The differences between the AUC in the GTV- and GPTV-based deep learning models were statistically significant (DeLong's test: $\mathrm{P}<0.01)$, indicating that the accuracy of the classification model improved by adding peritumor information. The corresponding ROC curves are shown in Figure 3.

\section{Discussion}

The deep learning model we establish here can be used to discriminate non-invasive adenocarcinoma from IAC both automatically and precisely. The model performs better identification when combined with peritumoral tissue analysis, compared with simple tumor tissue analysis. To our knowledge, this is the first study to evaluate the invasiveness of GGNs by considering tumor and peritumoral lung parenchyma based on preoperative CT images.

Accurate preoperative evaluation of the invasiveness of sub-solid nodules is imperative to choose the proper treatment strategy and evaluate prognosis. Pre-invasive lesions and MIA often have slow growth, long-term stability, or good prognosis, and can be clinically observed without surgery for a period of time. However, surgical treatment is urgently needed for invasive adenocarcinoma. Additionally, the prognosis of lung adenocarcinoma varies significantly among different pathological subtypes. Therefore, evaluation of GGN invasiveness is important for determining the appropriate clinical strategy.

Traditional morphological features of the tumor are used to judge the invasiveness of lung adenocarcinoma, including lesion size, density and lobulation sign (9,25-27). However, many nodules have atypical characteristics, and many junior doctors may be prone to make misdiagnosis due to subjective factors and a lack of understanding of GGNs. With the development of computer technology, certain quantitative parameters and radiomics have proved to be helpful in assessing the invasiveness of lung adenocarcinoma (28). However, they also have limitations, including a tedious workflow, time-consuming operation time and poor repeatability. In addition, uniform quantitative parameters do not exist across different research institutions. Thus, automatic, accurate and noninvasive alternative techniques are needed. With the advancement of computer vision technology, deep learning and convolution neural networks (CNNs) have been applied to some pathological detection and classification tasks. Machine learning tools have only been used to evaluate GGN invasiveness in a few studies $(29,30)$. However, most of these studies focused mostly 


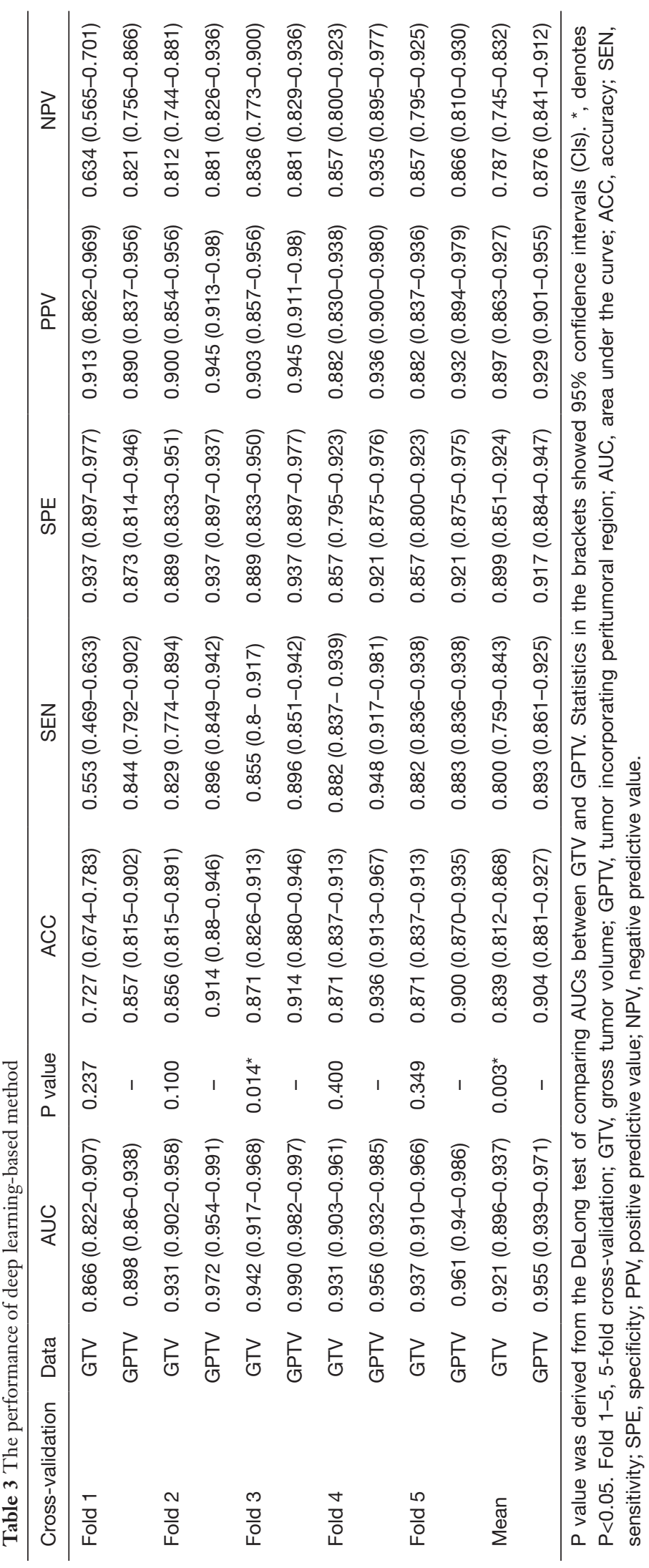



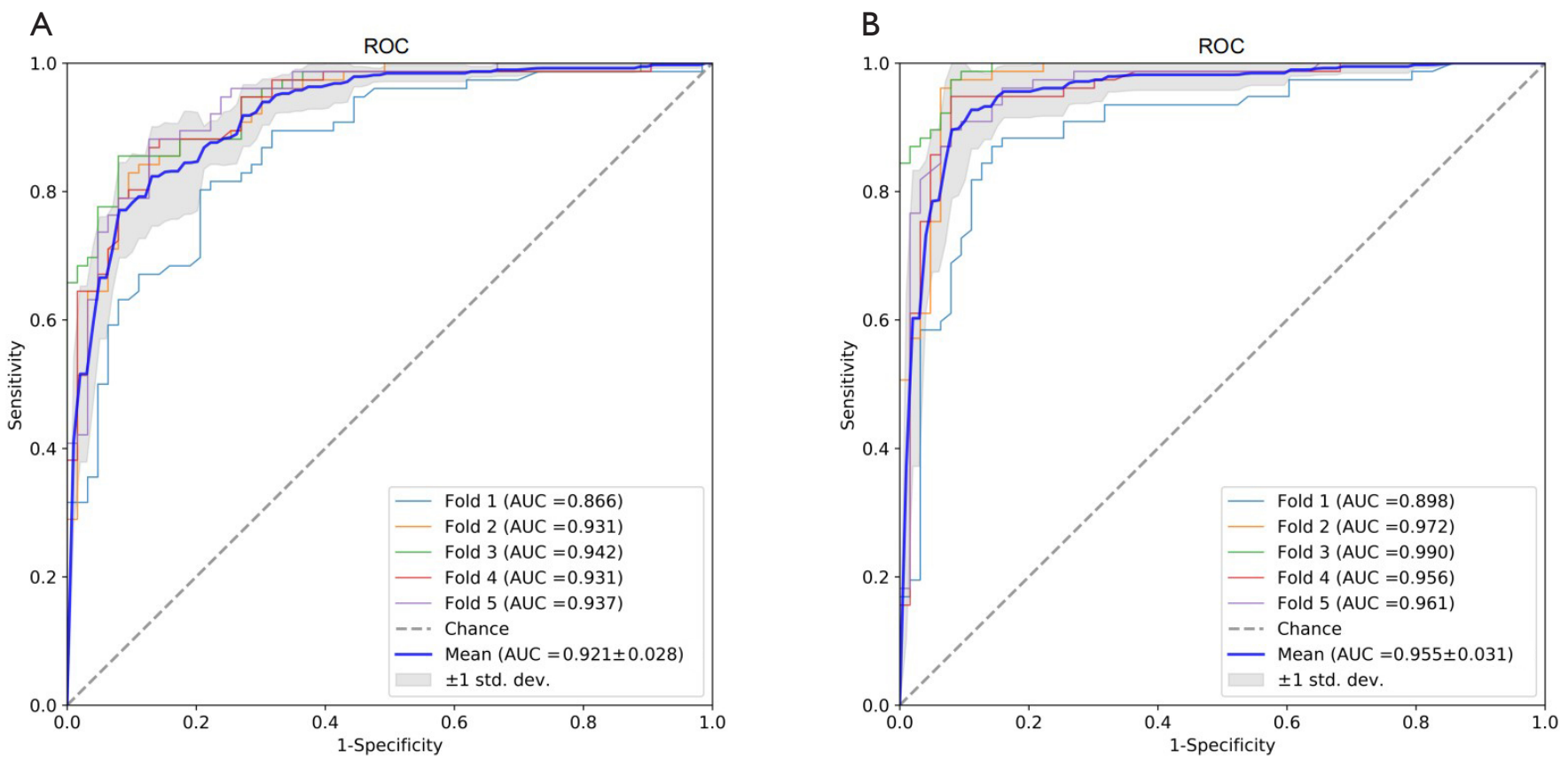

Figure 3 The receiver operating characteristic (ROC) curves using two types of inputs (A: GTV, B: GPTV). GTV, gross tumor volume; GPTV, tumor incorporating peritumoral region.

on the tumor area, ignoring the imaging features of the peritumoral lung parenchyma.

The tumor microenvironment plays a complex role in regulating tumor growth, infiltration and metastasis. The presence of peritumoral interstitial thickening, determined as a semantic feature on CT images, positively correlated with lymphovascular tumor invasion and tumor recurrence in NSCLC patients. Furthermore, Beig et al. (22) revealed that peritumoral radiomic features could significantly distinguish lung adenocarcinoma from lung granuloma, and the classification accuracy of this model was better than that of radiologists. Combined evaluation of peritumoral and tumoral radiomic features could increase the ability for accurate prediction of LN status (20). Peritumoral radiomic analyses have been widely used in other cancer types. For example, radiomic features from the peritumoral brain zone in routine pre-operative MRI scans may be predictive of postoperative recurrence in glioblastoma (31). This prediction model could be used to help surgeons make operation and treatment plans. Takada et al. showed that the radiomic profiles of peritumoral regions in MRI images could improve prognostic predictions (19). To the best of our knowledge, our study is the first to consider analyzing peritumoral lung parenchyma alongside tumoral characteristics in predicting GGN invasiveness.

Our findings support the idea that deep learning systems, built by combining GTV and PTV, could obtain the maximum performance in automated prediction of the invasiveness of lung adenocarcinoma manifesting as GGNs. Several articles report the ability of the model to predict the degree of pathological invasiveness with deep learning. Zhao et al. (29) demonstrated that the deep learning model could achieve better classification performance than the radiologists model, with an AUC of 0.880. A more recent study in 2020 demonstrated that the deep learning model could effectively differentiate invasive pulmonary adenocarcinomas that present as subsolid nodules, with an AUC of 0.921 (30). Here, we show that the AUC value of the model in the validation set increased from 0.921 to 0.955 with the addition of peritumor information. Thus, the PTV likely plays an important role in predicting tumor invasiveness. No previous studies have focused on the peritumoral microenvironments. The AUC of our deep learning model is higher than that in the two abovementioned articles. The PTV features included in our model may account for the higher AUC observed in 
our study. Additionally, the addition of PTV image features enhanced the baseline deep learning model and enabled differentiation of IAC from non-invasive lesions.

Our model can accurately predict the invasiveness of GGNs, improving the efficiency of lung cancer screening and assisting the efficiency of radiologists' work. The automatic segmentation method will soon be used with fully convolutional networks. GGN invasiveness can soon be calculated automatically through CT images. This model will provide a theoretical basis for individualized and precision treatment of patients with GGNs.

The findings of our study have several limitations. This is a single center study and therefore lacks an external validation set; Nevertheless, five CT scanners were used in our study and the deep learning classification model still shows good generalization ability across scanners. Further multicenter studies should be conducted to confirm our results. Second, we obtained a high level of predictive ability by combining tumoral with peritumoral lung parenchyma from preoperative CT images. However, the effect of peritumor size on invasive classification ability has not been proved. Finally, emphysema and interstitial lung disease may influence peritumoral lung parenchyma. Therefore, further studies should evaluate the effects of smokingrelated diffuse lung disease on the accuracy of our model.

\section{Conclusions}

Our results demonstrate that the tumor and peritumoral lung parenchyma of preoperative CT images show the best performance in predicting the invasiveness of GGNs when assessed together. Assessing the peritumoral lung parenchyma on preoperative CT images could enhance the ability to predict the invasiveness compared with a baseline model. This model can be used as an effective noninvasive prediction tool, which may aid decision making and defining an individualized and accurate medical treatment for patients with GGNs.

\section{Acknowledgments}

Funding: National Natural Science Foundation of China (Grant number 82001812); Contract grant sponsor: Pyramid Talent Project of Shanghai Changzheng Hospital; Shanghai Municipal Commission of Health and Family Planning Program (grant number 2018ZHYL0101); National Key Research and Development Project (grant number 2018YFC0116404); National Natural Science Foundation of China (grant number 81871321); National Key R\&D Program of China (grant number 2017YFC1308703).

\section{Footnote}

Reporting Checklist: The authors have completed the MDAR reporting checklist. Available at http://dx.doi.org/10.21037/ jtd-20-2981

Data Sharing Statement: Available at http://dx.doi. org/10.21037/jtd-20-2981

Peer Review File: Available at http://dx.doi.org/10.21037/jtd20-2981

Conflicts of Interest: All authors have completed the ICMJE uniform disclosure form (available at http://dx.doi. org/10.21037/jtd-20-2981). The authors have no conflicts of interest to declare.

Ethical Statement: The authors are accountable for all aspects of the work in ensuring that questions related to the accuracy or integrity of any part of the work are appropriately investigated and resolved. All procedures performed in this study were in accordance with the Declaration of Helsinki (as revised in 2013) and approved by the Ethics Committee of the Changzheng Hospital, Naval Medical University (No. 2018SL049), and individual consent for this retrospective analysis was waived.

Open Access Statement: This is an Open Access article distributed in accordance with the Creative Commons Attribution-NonCommercial-NoDerivs 4.0 International License (CC BY-NC-ND 4.0), which permits the noncommercial replication and distribution of the article with the strict proviso that no changes or edits are made and the original work is properly cited (including links to both the formal publication through the relevant DOI and the license). See: https://creativecommons.org/licenses/by-nc-nd/4.0/.

\section{References}

1. Huang KL, Wang SY, Lu WC, et al. Effects of lowdose computed tomography on lung cancer screening: a systematic review, meta-analysis, and trial sequential analysis. BMC Pulm Med 2019;19:126.

2. National Lung Screening Trial Research T, Aberle DR, Adams AM, et al. Reduced lung-cancer mortality with 
low-dose computed tomographic screening. N Engl J Med 2011;365:395-409.

3. Sakurai H, Dobashi Y, Mizutani E, et al. Bronchioloalveolar carcinoma of the lung 3 centimeters or less in diameter: a prognostic assessment. Ann Thorac Surg 2004;78:1728-33.

4. Jia M, Yu S, Cao L, et al. Clinicopathologic Features and Genetic Alterations in Adenocarcinoma In Situ and Minimally Invasive Adenocarcinoma of the Lung: LongTerm Follow-Up Study of 121 Asian Patients. Ann Surg Oncol 2020;27:3052-63.

5. Sagawa M, Oizumi H, Suzuki H, et al. A prospective 5 -year follow-up study after limited resection for lung cancer with ground-glass opacity. Eur J Cardiothorac Surg 2018;53:849-56.

6. Yanagawa N, Shiono S, Abiko M, et al. New IASLC/ATS/ ERS classification and invasive tumor size are predictive of disease recurrence in stage I lung adenocarcinoma. J Thorac Oncol 2013;8:612-8.

7. Eguchi T, Kadota K, Park BJ, et al. The new IASLCATS-ERS lung adenocarcinoma classification: what the surgeon should know. Semin Thorac Cardiovasc Surg 2014;26:210-22.

8. Sakurai H, Nakagawa K, Watanabe S, et al. Clinicopathologic features of resected subcentimeter lung cancer. Ann Thorac Surg 2015;99:1731-8.

9. Ding H, Shi J, Zhou X, et al. Value of CT Characteristics in Predicting Invasiveness of Adenocarcinoma Presented as Pulmonary Ground-Glass Nodules. Thorac Cardiovasc Surg 2017;65:136-41.

10. Fan L, Fang M, Li Z, et al. Radiomics signature: a biomarker for the preoperative discrimination of lung invasive adenocarcinoma manifesting as a ground-glass nodule. Eur Radiol 2019;29:889-97.

11. Li Q, Fan L, Cao ET, et al. Quantitative CT analysis of pulmonary pure ground-glass nodule predicts histological invasiveness. Eur J Radiol 2017;89:67-71.

12. Esteva A, Kuprel B, Novoa RA, et al. Dermatologist-level classification of skin cancer with deep neural networks. Nature 2017;542:115-8.

13. Chassagnon G, Vakalopoulou M, Paragios N, et al. Artificial intelligence applications for thoracic imaging. Eur J Radiol 2020;123:108774.

14. Liu Y, Balagurunathan Y, Atwater T, et al. Radiological Image Traits Predictive of Cancer Status in Pulmonary Nodules. Clin Cancer Res 2017;23:1442-9.

15. Ragab DA, Sharkas M, Marshall S, et al. Breast cancer detection using deep convolutional neural networks and support vector machines. PeerJ 2019;7:e6201.

16. Valencia T, Kim JY, Abu-Baker S, et al. Metabolic reprogramming of stromal fibroblasts through p62mTORC1 signaling promotes inflammation and tumorigenesis. Cancer Cell 2014;26:121-35.

17. Zhang B, Yao G, Zhang Y, et al. M2-polarized tumorassociated macrophages are associated with poor prognoses resulting from accelerated lymphangiogenesis in lung adenocarcinoma. Clinics (Sao Paulo, Brazil) 2011;66:1879-86.

18. Faget J, Groeneveld S, Boivin G, et al. Neutrophils and Snail Orchestrate the Establishment of a Protumor Microenvironment in Lung Cancer. Cell Rep 2017;21:3190-204.

19. Takada A, Yokota H, Watanabe Nemoto M, et al. A multi-scanner study of MRI radiomics in uterine cervical cancer: prediction of in-field tumor control after definitive radiotherapy based on a machine learning method including peritumoral regions. Jpn J Radiol 2020;38:265-73.

20. Wang X, Zhao X, Li Q, et al. Can peritumoral radiomics increase the efficiency of the prediction for lymph node metastasis in clinical stage T1 lung adenocarcinoma on CT? Eur Radiol 2019;29:6049-58.

21. Lantuejoul S, Rouquette I, Brambilla E, et al. New WHO classification of lung adenocarcinoma and preneoplasia. Ann Pathol 2016;36:5-14.

22. Beig N, Khorrami M, Alilou M, et al. Perinodular and Intranodular Radiomic Features on Lung CT Images Distinguish Adenocarcinomas from Granulomas. Radiology 2019;290:783-92.

23. Wu G, Woodruff HC, Shen J, et al. Diagnosis of Invasive Lung Adenocarcinoma Based on Chest CT Radiomic Features of Part-Solid Pulmonary Nodules: A Multicenter Study. Radiology 2020;297:E282.

24. DeLong ER, DeLong DM, Clarke-Pearson DL. Comparing the areas under two or more correlated receiver operating characteristic curves: a nonparametric approach. Biometrics 1988;44:837-45.

25. Godoy MC, Sabloff B, Naidich DP. Subsolid pulmonary nodules: imaging evaluation and strategic management. Curr Opin Pulm Med 2012;18:304-12.

26. Yu X, Li Y, Shi C, et al. Risk factors of lymph node metastasis in patients with non-small cell lung cancer $</=$ $2 \mathrm{~cm}$ in size: A monocentric population-based analysis. Thorac Cancer 2018;9:3-9.

27. Lee SM, Park CM, Goo JM, et al. Invasive pulmonary adenocarcinomas versus preinvasive lesions appearing as 
ground-glass nodules: differentiation by using CT features. Radiology 2013;268:265-73.

28. Chen X, Fang M, Dong D, et al. A Radiomics Signature in Preoperative Predicting Degree of Tumor Differentiation in Patients with Non-small Cell Lung Cancer. Acad Radiol 2018;25:1548-55.

29. Zhao W, Yang J, Sun Y, et al. 3D Deep Learning from CT Scans Predicts Tumor Invasiveness of Subcentimeter Pulmonary Adenocarcinomas. Cancer Res 2018;78:6881-9.

30. Kim H, Lee D, Cho WS, et al. CT-based deep learning

Cite this article as: Wang $X$, Chen K, Wang W, Li Q, Liu K, Li Q, Cui X, Tu W, Sun H, Xu S, Zhang R, Xiao Y, Fan L, Liu S. Can peritumoral regions increase the efficiency of machinelearning prediction of pathological invasiveness in lung adenocarcinoma manifesting as ground-glass nodules? J Thorac Dis 2021;13(3):1327-1337. doi: 10.21037/jtd-20-2981 model to differentiate invasive pulmonary adenocarcinomas appearing as subsolid nodules among surgical candidates: comparison of the diagnostic performance with a sizebased logistic model and radiologists. Eur Radiol 2020;30:3295-305.

31. Prasanna P, Patel J, Partovi S, et al. Radiomic features from the peritumoral brain parenchyma on treatment-naive multi-parametric MR imaging predict long versus shortterm survival in glioblastoma multiforme: Preliminary findings. Eur Radiol 2017;27:4188-97. 
Supplementary

Table S1 Relationship between Peritumoral CT Feature and pathological invasiveness of GGNs

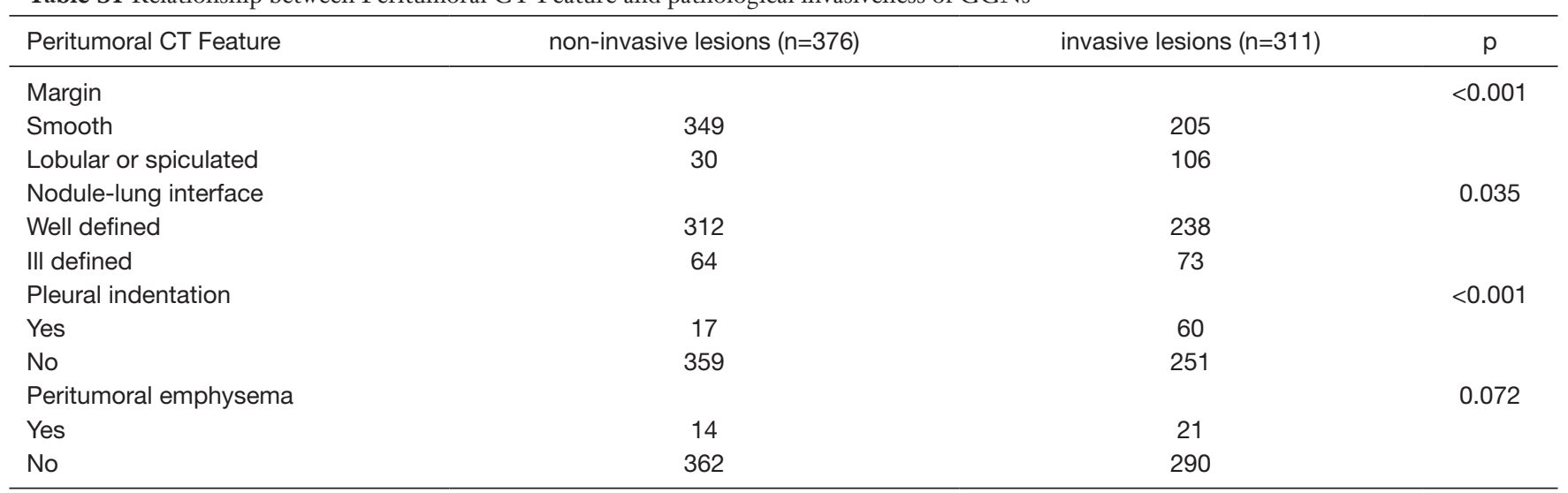

